\title{
Location of air-borne SAR imagery without GCPs and error analysis
}

\author{
FAN Ning-jun, ZHAO Ling-jun, KUANG Gang-yao, CUI Ying \\ National University of Defense Technology \\ Changsha, Hunan, China
}

\begin{abstract}
A latitude-longitude positioning-method has been developed to determine the location of air-borne SAR(Synthetic Aperture Radar) imagery without GCPs(Ground Control Points) based on the F.Leberl Model by using GPS(Global Positioning System)/INS(Inertial Navigation System) system and errors caused by the system are analyzed. By using the measurement-data of the GPS/INS system, the geographical coordinate value of the target can be gotten by combining the three equations: SAR Range Equation, SAR Zero-Doppler Equation and SAR Height Equation. Then the latitude-longitude value of the target can be calculated. This method has an advantage over previous methods in that the iterative algorithm which has to choose a initial point and the roll/pitch of the plane need not to be considered. The numerical experimental result demonstrated the validity of the positioning-method, and depending on the result of the error analysis, some useful applications are recommended.
\end{abstract}

Index Terms - Air-borne SAR, GPS/INS, Location without GCPs, Latitude-longitude Location.

\section{INTRODUCTION}

Location of target in SAR image is the fundamental information when we want to make full use of SAR imagery. Thus how to get precise location information of target in SAR image is an important and hot section in the research of remote sensing[1, 2].

Traditional locating-method for SAR image is generally using GCPs. However, there are many places which are difficult to deploy GCPs such as deserts and seas. Thus, locating-method without GCPs is very useful. And in order to make good use of the location information, it is usually represented as latitude-longitude value[3].

F.Leberl Model uses the geometric characteristics of SAR imagery to locating pixel on Earth[4]. This model is simple and uses SAR-imagery mechanism. The possibility to use F.Leberl Model for SAR-location without GCPs was explained in Paper [5], but it was not practical for the lack of position-attitude technique of airplane at that time.

As the booming development of GPS and INS applied to position-attitude measurement for airplane, the GPS/INS can get airplane's velocity, position and attitude information quickly and precisely. Thus, F.Leberl Model for location without GCPs is becoming implementable. Thus, a latitude-longitude locating-method without GCPs based on F.Leberl Model and the corresponding flow are developed. And the errors caused by GPS/INS are analyzed. At last, some useful applications are recommended.

\section{THEORY}

Leberl developed the radar collinear equation on the ITC Technique Report, which is now named as F.Leberl Model. Let $R_{S}$ as the distance between radar sensor $S$ and target $P$ on ground,

$$
R_{S}^{2}=\left(X-X_{S}\right)^{2}+\left(Y-Y_{S}\right)^{2}+\left(Z-Z_{S}\right)^{2}
$$

$S$ is the position of the radar sensor, $(X, Y, Z)$ is the coordinate value of target $P$ on the ground, $H$ is the height of the radar sensor, $\left(X_{S}, Y_{S}, Z_{S}\right)$ is the coordinate value of the real-time position of the radar sensor $S$.

Under the condition that the side-glance angle of beam is zero, the Doppler frequency is zero. Let $\left(\dot{X}_{S}, \dot{Y}_{S}, \dot{Z}_{S}\right)$ as the real-time velocity of the SAR-platform, then

$$
\dot{X}_{S}\left(X-X_{S}\right)+\dot{Y}_{S}\left(Y-Y_{S}\right)+\dot{Z}_{S}\left(Z-Z_{S}\right)=0
$$

The equation (1) and equation (2) are so called the F.Leberl Model of airborne SAR imagery. And equation (1) is called the Range Equation, equation (2) is called the Zero-Doppler Equation.

For the equation (1) and (2), $R_{S}$ can be calculated from the back-wave data by using the Range-measuring Method[6]. When having selected the coordinate system, $\left(X_{S}, Y_{S}, Z_{S}\right)$ 
and $\left(\dot{X}_{S}, \dot{Y}_{S}, \dot{Z}_{S}\right)$ can be got by the measuring-data of the GPS/INS system.

Table 1 represents the measurement accuracy of POS AV510 system[7]. RT DGPS represents the accuracy of real-time differential data, And Post Processed represents the accuracy of the post processed differential data. From Table 1, the real-time positioning accuracy is $0.5-2.0 \mathrm{~m}$, the real-time velocity accuracy is $0.05 \mathrm{~m} / \mathrm{s}$. The accuracy can satisfy lots of positioning-applications. Thus, this GPS/INS system is used to test the positioning-method in this paper.

\begin{tabular}{|c|c|c|}
\hline & RT DGPS & Post Processed \\
\hline Positon (m) & $0.5 \sim 2.0$ & $0.05 \sim 0.30$ \\
\hline Velocity $(\mathrm{m} / \mathrm{s})$ & 0.05 & 0.005 \\
\hline Roll/Pitch (deg) & 0.008 & 0.005 \\
\hline Heading (deg) & 0.050 & 0.008 \\
\hline
\end{tabular}

\section{III.POSITIONING METHOD}

Using F.Leberl Model for latitude-longitude positioning, the following issues must be solved: firstly, in order to obtaining the value of $(X, Y, Z)$, the third equation must be added. Secondly, a simple but convenient coordinate system should be selected. Thirdly, the result should be represented as latitude-longitude style.

\section{A. Height Equation}

Generally, for airborne SAR, the curvature can be ignored when the radius of the imagery region is within $30 \mathrm{~km}$, and directly let the spherical surface as the plane surface as Paper [8] demonstrated.

As the radius of airborne SAR imagery section is within $30 \mathrm{~km}$, the section can be treated as plane surface. What's more, the flying-height of SAR-platform is $3000-5000 \mathrm{~m}$ in general, incidence angle is between 30-80 degrees, thus the distance between the projection of radar sensor and the corresponding target is within $30 \mathrm{~km}$ generally. Supposing that the flying-height of SAR-platform is as high as $10,000 \mathrm{~m}$, the incidence angle is as large as 80 degrees, the distance between the projection of radar sensor with the corresponding target is $28.794 \mathrm{~m}$ which is less than $30 \mathrm{~km}$. So the curvature can be ignored when dealing with positioning-method of airborne SAR imagery.

\section{B. The coordinate system}

Considering that the velocity of SAR-platform is generally presented with geographical coordinate system, we select it as the fundamental coordinate system for simple and convenient. The coordinate system is as follows: the radar sensor of SAR-platform is the origin, the East orientation is the direction of $x$ axis, the North orientation is the direction of $y$ axis and $z$ axis is vertical with $x$ axis as well as $y$ axis to form a right-hand coordinate system, which is called as East-North-Sky System.

From the previous analysis, the curvature can be ignored in the East-North-Sky System. Let $H$ as the height of the platform and $h$ as the height of the target, then

$$
Z-Z_{S}=-(H-h)
$$

$H$ can be measured by the POS AV510 system; Suppose that the imagery-section on the ground is plan, then $h$ can be taken as the average of the height of the imagery-section.

By using the Range Equation, Zero-Doppler Equation and Height Equation, the target's geographical coordinate value can be calculated. And by using the coordinate transformation, the geographical coordinate can be transformed to latitude-longitude representation, which is the wanted answer.

C. Calculating the target's geographical coordinate value

Combine formula (1), (2) and (3), and notice that the origin of the coordinate is the radar sensor, so the velocity of radar sensor is 0 . That is to say $X_{S}=Y_{S}=Z_{S}=0$, then the formula will be

$$
\left\{\begin{array}{l}
X^{2}+Y^{2}+Z^{2}=R_{S}{ }^{2} \\
\dot{X}_{S} X+\dot{Y}_{S} Y+\dot{Z}_{S} Z=0 \\
Z=h-H
\end{array}\right.
$$

As for formula (4), the radar sensor's velocity $\left(\dot{X}_{S}, \dot{Y}_{S}, \dot{Z}_{S}\right)$ and height $H$ can be measured by the POS AV510 system, the range $R_{S}$ can be calculated by some corresponding method, the height $h$ of the target on the ground is a known value. In this equation set, there are three independent equations and three independent unknown parameters. So the unknown parameters can be calculated, which is the position-value of the target in the SAR image.

By solving equation(4), we get 


$$
Y=\frac{-\dot{Y}_{S} \square\left(\dot{Z}_{S} Z\right) \pm A}{\left(\dot{X}_{S}^{2}+\dot{Y}_{S}^{2}\right)}
$$

And

$$
A=\sqrt{\dot{X}_{S}^{2}\left[\left(\dot{X}_{S}^{2}+\dot{Y}_{S}^{2}\right)\left(R_{S}^{2}-Z^{2}\right)-\dot{Z}_{S}^{2} \square Z^{2}\right]}
$$

From equation(5), the exact $Y$ has to be determined. Firstly, suppose that the two keys of $Y$ are $Y_{1}$ and $Y_{2}$, then $Y_{1} \square Y_{2}=\frac{\dot{Z}_{S}^{2} \square Z^{2}-\dot{X}_{S}^{2}\left(R_{S}{ }^{2}-Z^{2}\right)}{\dot{X}_{S}{ }^{2}+\dot{Y}_{S}{ }^{2}}$. In this equation, the denominator is positive, considering the symmetrical characteristics of $\dot{X}_{S}$ and $\dot{Y}_{S}$, let $\dot{X}_{S} \geq \dot{Y}_{S}$. Under the condition of the radar sensor's velocity equal to $100 \mathrm{~m} / \mathrm{s}$, $\dot{X}_{S} \geq 70$. Generally, the vertical velocity $\dot{Z}_{S}$ is very small, $\dot{Z}_{S} \leq 10$, then $49 \dot{Z}_{S}{ }^{2} \leq \dot{X}_{S}{ }^{2}$. What's more, $Z^{2}$ means the square of the distance between the radar sensor and the target on the ground in $z$ direction, $R_{S}{ }^{2}-Z^{2}$ represents the square of the distance between the projective point of the radar sensor and the target on the ground. As the incidence angle is within $30-80$ degrees in general, $Z^{2} \leq 2\left(R_{S}{ }^{2}-Z^{2}\right)$. So $\dot{Z}_{S}{ }^{2} \square Z^{2} \leq \dot{X}_{S}{ }^{2}\left(R_{S}{ }^{2}-Z^{2}\right)$, which means the numerator is negative. Thus, $Y_{1} \square Y_{2}<0$. The two keys have opposite signs. Secondly, the sign of $Y$ has to be determined. Suppose the illuminating-direction of the radar beam is within the first and second quadrant of xoy plane, $Y$ is positive. Otherwise, $Y$ is negative. In the test of this paper, the East and North velocity is positive. Then the illuminating direction of radar beam is within the fourth quadrant, and the target is in this section. So $Y$ is negative.

By using the above method, the value of $Y$ can be calculated. Combining equation(4), the geography-position value $(X, Y, Z)$ of the target can be gotten. Then by employing the transformation-method between the geographical coordinate and the Earth-based coordinate of Paper [9], the Earth-based coordinate value $\left(x_{e}, y_{e}, z_{e}\right)$ and the latitude-longitude value of the target.can be gotten.

\section{Positioning flow}

The latitude-longitude positioning method has two steps: firstly, combine the Range Equation, Zero-Doppler Equation and Height Equation to obtain the geographical coordinate value of the target. Secondly, transform the geographical coordinate value to latitude-longitude value (Figure 1).

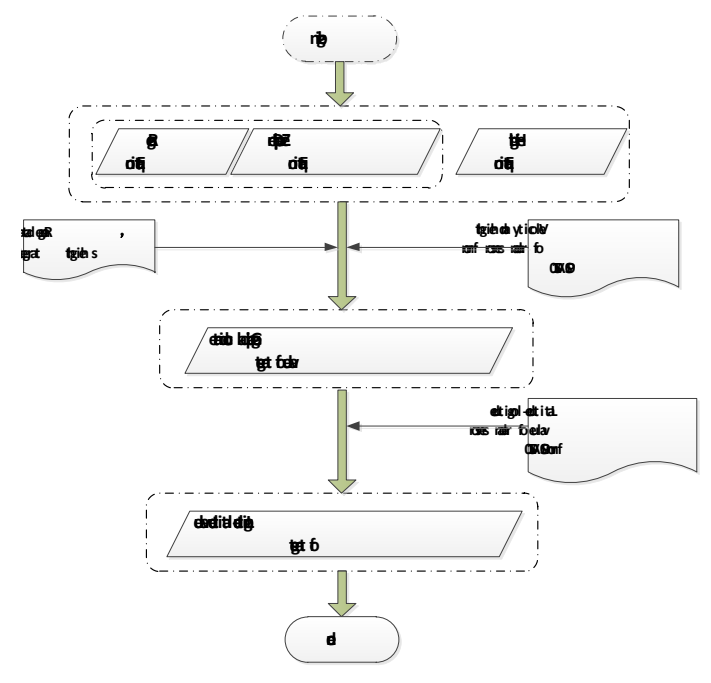

Figure 1 positioning flow

\section{ERROR ANALYSIS}

In order to make the error analysis clearly, the measurement data of GPS/INS system is separated into three parts: velocity data, position data and the total data. As the positioning-method is based on the reference between the target and the radar sensor, the error of position data will add to the target's positioning-value linearly. What's more, the total error caused by GPS/INS system is made up of velocity measured-error and position measured-error of the radar sensor. So we just need to analysis the velocity measured-error. We test the result for 1000 times and the parameter-setting is referred to the POS AV510 system as presented by Table 1 .

The velocity of the radar sensor is made up of three parts: the East velocity, the North velocity and the vertical velocity. The velocity is generally on the order of hundred. In our test, the velocity is $100 \mathrm{~m} / \mathrm{s}$, the flying-direction is North-East, so the East and North velocity are both $70 \mathrm{~m} / \mathrm{s}$, and the ideal vertical velocity is zero. Considering the randomness of the GPS/INS measure-system, the measured-velocity follows the Gaussian distribution with $5 \sigma$ criterion presented by Table 2 . 
Other parameters are presented by Table 3.

Table 2 the value of the three velocity of the radar sensor

\begin{tabular}{|l|l|l|l|}
\hline & Ideal value & accuracy & distribution \\
\hline $\begin{array}{l}\text { East } \\
\text { velocity }\end{array}$ & $70 \mathrm{~m} / \mathrm{s}$ & $0.05 \mathrm{~m} / \mathrm{s}$ & $\mathrm{N}\left(70,0.01^{2}\right)$ \\
\hline $\begin{array}{l}\text { North } \\
\text { velocity }\end{array}$ & $70 \mathrm{~m} / \mathrm{s}$ & $0.05 \mathrm{~m} / \mathrm{s}$ & $\mathrm{N}\left(70,0.01^{2}\right)$ \\
\hline $\begin{array}{l}\text { Vertical } \\
\text { velocity }\end{array}$ & 0 & $0.05 \mathrm{~m} / \mathrm{s}$ & $\mathrm{N}\left(70,0.01^{2}\right)$ \\
\hline
\end{tabular}

Table 3 the value of other parameters

\begin{tabular}{|l|l|l|}
\hline Range value & Side-glance angle & Flying-height \\
\hline $10000 \mathrm{~m}$ & 0 & $5000 \mathrm{~m}$ \\
\hline $\begin{array}{l}\text { Longitude of } \\
\text { radar sensor }\end{array}$ & $\begin{array}{l}\text { Latitude of radar } \\
\text { sensor }\end{array}$ & $\begin{array}{l}\text { Target's height on } \\
\text { the ground }\end{array}$ \\
\hline E 112 & N 28 & $50 \mathrm{~m}$ \\
\hline
\end{tabular}

average of absolute positioning-еггог: 25.7162

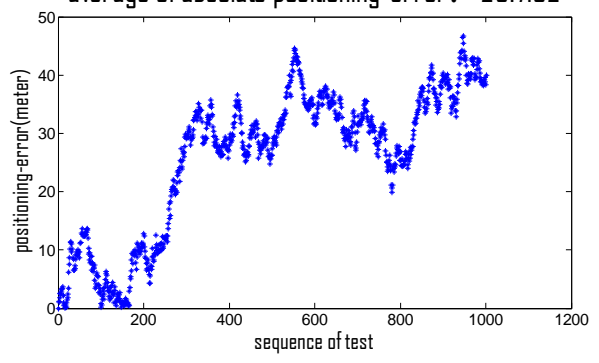

(a) the specific positioning-error in each line
The test result is presented by Figure 2(a) and Figure 2(b). From the result of the test: the average of the absolute positioning-error (the distance between the test position and the real position of the target on the ground) is 25.7162 meters, the largest error is less than 50 meters. And the positioning-error is widely distributed within 20-40 meters. The positioning-error of POS AV510 system is 0.5-2.0 meters presented as Table 1, so the average positioning-error of this method is about $25-30$ meters, the largest positioning-error is less than 60 meters. And the total positioning-error is widely distributed within20-40 meters.

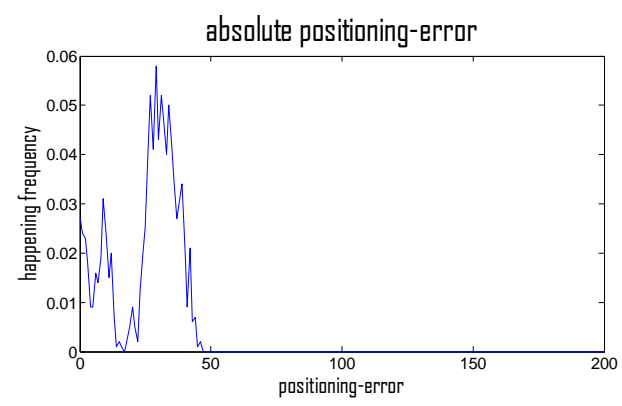

(b) the happening frequency of each positioning-error

Figure 2 the experimental results

\section{CONCLUSION}

A latitude-longitude positioning-method of SAR imagery based on the F.Leberl Model by using the GPS/INS system is developed and the corresponding operating flow is given. This method has a widely utilization and when the flying-velocity is not in parallel with the horizontal plane, this method can still work. What's more, by using this method, we need not to consider the iterative algorithm, which needs to choose an initial point and the roll/pitch of the plane. And it directly exports longitude-latitude value of pixels on the SAR image. From the test result, when using POS AV510 system and employing the Gaussian distribution with $5 \sigma$ criterion, the average positioning-error is about 27 meters, the largest positioning-error is less than 60 meters and the total positioning-error is within 20-40 meters. In military awareness, dynamic supervision for natural disaster, sea monitoring, source searching and so on, the happening-section can be determined rapidly, and then take some corresponding measures.

\section{REFERENCES}

[1] S. Y. YAN Lei, "Analysis of location precision in TerraSAR image without reference points," Computer Engineering and Applications, vol. 47, pp. 174-176, 2011.

[2] H. B. Liu Jiayin, Hong Wen, "A New Direct Solution of Range-doppler Model for SAR Image," Remote Sensing Technology And Application, vol. 27, pp. 716-721, 2012.

[3] L. Y.-q. PENG Dai-qiang, DU Peng-fei, "A Fast Algorithm for Latitude and Longitude Calculation of Airborne SAR and Its Location Precision Analysis," Modern Radar, vol. 32, pp. 48-53, 2010.

[4] F.Leberl, "Radargrammetry for Image 
Interpretation," presented at the ITC Technical Report, 1978.

[5] X. Q. ZHU Cai-ying, WU Cong-hui, CHI Tian-he, HE Jian-bang, "Study on Mathematical Models for Airborne SAR Image Rectification," Journal of Remote Sensing, vol. 7, pp. 112-117, 2003.

[6] C. A. SUN Wen-feng, DENG Hai-tao, YU Gen-miao, "A Novel Geometric Calibration and Target Location Algorithm for Air-borne SAR Image," Acta Electronica Sinica, vol. 35, pp. 553-556, 2007.

[7] C. Li-fu, "Research on Airborne Dual-antenna InSAR Real-time Processing Algorithm,"
DOCTOR, Chinese Academy of Sciences, Chinese Academy of Sciences, Beijing, 2010.

[8] D. C. YOU Hong-jian, XIANG Maosheng, "Principle and Accuracy for Airborne SAR Image Direct Localization," Geomatics and Information Science of Wuhan University, vol. 30, pp. 712-715, 2005.

[9] S. W.-f. YAO Han-ying, QIAN Li-chang, "A Method of Absolute Positioning of Air-borne Strip SAR Image," Journal of Air Force Radar Academy, vol. 23, pp. 257-260, 2009. 